\title{
INFLUENCIA DE LOS FACTORES DE LA MADERA Y DE LAS CONDICIONES DEL MACERADO EN EL TIEMPO DE CALENTAMIENTO DE TROZAS
}

\author{
Rogelio Moreno Muñoz (*) \\ Francis Devlieger Sollier (**)
}

\section{RESUMEN}

En el presente trabajo se expone una modelación lineal de conducción de calor transiente para predecir tiempo de calentamiento de rollizos de madera hasta la temperatura deseada para el debobinado.

El modelo se resuelve por aproximación numérica, empleando el método explícito de las diferencias finitas.

Se analiza también la influencia que ejerce la anisotropía de la madera en los tiempos de calentamiento.

Los resultados muestran que para relaciones superiores a 3:1 entre el largo y el diámetro de la troza, el tiempo de calentamiento no se ve influenciado por la anisotropia de la madera. El tiempo de calentamiento depende fundamentalmente del diámetro de la troza a considerar, como asimismo del contenido de humedad de la troza y del gradiente de temperatura de la madera y del baño.

Palabras claves: Macerado de Trozas, Transferencia de Calor, Método Computacional.

\section{ABSTRACT}

This paper presents a linear model of transient heat conduction to estimate log heating times for a given log peeling temperature.

The model solves for a numeric approximation using an explicit finitedifference technique.

The wood anistropic effect on heating times is evaluated. It is found that for a relation 3:1 or more between log length and diameter, the anisotropic effect on heating time can be omitted.

The heating time depends fundamentally on log diameter, moisture content and gradient between wood and bath temperatures.

Key words : Log heating - heat conduction for log - computer program for log heating times.

\footnotetext{
* Ingeniero Civil Mecánico, Master en Ciencias, Ingenieria Mecánica, Instituto de Materiales y Procesos Termomecánicos. Facultad de Ciencias de la Ingeniería, Universidad Austral de Chile. Casilla 567 Valdivia.

** Ingeniero de la Madera, Inst. Tecnologia de Productos Forestales, Facultad de Ciencias Forestales. Universidad Austral de Chile. Casilla 567 - Valdivia.
} 


\section{INTRODUCCION}

En la industria de contrachapados, se requiere de un calentamiento de las trozas de madera con el fin de ablandarlas para el posterior debobinado y elaboración de las chapas exentas de daños. El ablandamiento de los rollizos con el calor se efectúa en piletas de cocción, ya sea con vapor o agua caliente; trabajos de investigación indican que la rapidez del proceso en ambos casos es similar.

En la industria chilena, el proceso se realiza en piletas de agua caliente con tiempos de permanencia que debieran ser suficientes para calentar la madera a la temperatura adecuada hasta el rollizo central.

La temperatura al interior de la troza no es fácil de medir en terreno y se han publicado tablas para determinar tiempos de macerado; sin embargo, estas tablas se aplican a la madera del hemisferio norte y la información entregada es válida solamente para los procesos en condiciones similares a las investigadas por Feihl (1972) y Feihl y Godin (1975). La información teórica proporcionada por Steinhagen et al. (1980), Steinhagen (1986) y Steinhagen et al. (1987), no es aplicable a la industria sin un programa computacional que entregue rápidamente los tiempos de calentamiento. Además el modelo no toma en cuenta la anisotropía de la madera.

El objetivo de este trabajo consiste en estudiar modelos lineales de conducción de calor transiente, para simular el calentamiento y predecir el tiempo de macerado de las trozas en un baño de agua caliente a temperatura constante. Se analiza un modelo unidireccional de conducción radial y otro de conducción bidireccional, considerando la anisotropía de la madera en cuanto a su difusión térmica. Las hipótesis del trabajo consisten en considerar, por un lado, que la madera posee propiedades termicas constantes para valores medios de temperatura y contenido de humedad de las trozas durante el proceso y por otro que la temperatura inicial de la troza es uniforme y superior a $0{ }^{\circ} \mathrm{C}, \mathrm{y}$ la del baño constante.

La temperatura deseada en el rollizo central para el posterior debobinado, se deduce del trabajo de Lutz (1972), en función de la densidad básica de la especie a considerar. 


\section{FORMULACION DEL MODELO MATEMATICO}

La base matemática del trabajo es la ecuación de Fourier para conducción transiente en sólidos con propiedades térmicas y temperatura inicial constantes.

$$
\begin{aligned}
& \rho_{\mathrm{C}} \frac{\mathrm{T}}{\mathrm{t}}=\nabla \cdot\left[\mathrm{K}_{\mathrm{t}} \nabla \mathrm{T}_{\mathrm{r}}+\mathrm{k}_{\mathrm{z}} \nabla \mathrm{T}_{\mathrm{z}}\right] \\
& \frac{\partial \mathrm{T}}{\partial \mathrm{t}}=\nabla \cdot\left[\begin{array}{llll}
\alpha & \mathrm{T} \mathrm{e}_{t}+\alpha_{z} & \nabla & \mathrm{Te}_{2}
\end{array}\right]
\end{aligned}
$$

donde :

$$
\alpha=\frac{\mathrm{k}}{\rho_{\mathrm{C}}}
$$

Haciendo empleo de coordenadas cilíndricas, el modelo unidireccional a resolver es :

$$
\begin{aligned}
\frac{\partial T}{\partial t} & =\alpha_{r}\left(\frac{\partial^{2} T}{\partial r}+\frac{1}{r} \cdot \frac{\partial T}{\partial r}\right) ; r \neq 0 \\
-k_{r} \frac{\partial T}{\partial r} & =h\left(T-T_{b}\right) ; r=R \\
\frac{\partial T}{\partial r} & =0 ; \quad r=0 \\
T & =T_{i} ; \quad t=0
\end{aligned}
$$

En el caso bidireccional la ecuación diferencial es :

$$
\frac{\partial T}{\partial t}=\partial_{r}\left(\frac{\partial^{2} T}{\partial_{r 2}}+\frac{1}{r} \cdot \frac{\partial T}{\partial_{r}}\right)+\alpha_{2} \frac{\partial^{2} T}{\partial_{z}^{2}} ; r \neq 0
$$

Los valores de las propiedades térmicas de la madera, necesarios para desarrollar la ecuación de Fourier se correlacionaron con la humedad, temperatura y densidad básica de la misma, en base a los datos recopilados por Steinhagen (1977). Específicamente, la correlación para la difusión térmica radial es :

$$
\begin{aligned}
\alpha_{r} & =\left(\alpha_{0}+\mathrm{aT}\right) \mathrm{A}^{\alpha} \\
\alpha_{0} & =\left(5,58-1,08 \times 10^{-2} \mathrm{H}\right) \times 10^{-4} ; \mathrm{T}=0 \cdot \mathrm{C}, 40 \%<\mathrm{H}<120 \% \\
\mathrm{~A} & =1,15-0,29 \mathrm{GS}
\end{aligned}
$$


La constante "a" asume el valor $6 \times 10^{-7}$ para el rango de $40 \%<\mathrm{H}<80 \%$ y 8,5 $x 10^{-7}$ para el rango de $80 \%<\mathrm{H}<120 \%$

Al considerar el modelo bidireccional se asume una relación de 2:1, entre la conductividad axial y la conductividad radial, según se desprende del trabajo de Steinhagen (1977).

La evaluación del coeficiente de transferencia de calor por convección natural, se realizó empleando la ecuación de Mc Adams (1954).

$$
\mathrm{Nu}=\frac{\mathrm{hD}}{\mathrm{k}}=0,53\left[\frac{\mathrm{g} \beta\left(\mathrm{T}_{\mathrm{b}}-\mathrm{T}_{\mathrm{r}=\mathrm{R}}\right) \mathrm{D}^{3}}{\mathrm{v}^{2}} \mathrm{Pr}\right]^{0,25}
$$

Válida dentro del rango $10^{4}<\mathrm{GrPr}<10^{9}$

El modelo matemático se resuelve por aproximación numérica, empleando el método explícito de diferencias finitas, dando origen a las siguientes ecuaciones, para el campo de la temperatura, en el caso del modelo radial con una malla de $\mathrm{N}$ nodos.

$$
\begin{aligned}
& T_{i}{ }^{n+1}-\frac{1}{M}\left[T_{i-1}{ }^{n}+T_{i+1}{ }^{n}+\frac{T_{i+1}{ }^{n}-T_{i-1}{ }^{n}}{2 i}\right]+T_{i}^{n}\left[1-\frac{2}{M}\right] ; i=1,2, \ldots, N-1 \\
& M=\frac{\Delta r^{2}}{d_{r} \Delta t} \geqslant 2 \\
& T_{N}{ }^{n+1}=\frac{h T_{b}+\left(k_{r} T_{N-1}^{n+1} / \Delta r\right)}{h+k_{r} / \Delta r} \\
& T_{0}^{n+1}=\frac{4 T_{1}^{n}}{M}+T_{0}^{n}\left[1-\frac{4}{M}\right] ; i=0, M \geqslant 4
\end{aligned}
$$

El método también es aplicable al modelo que considera conducción radial y axial; sin embargo, la condición de estabilidad, en este caso, exige emplear intervalos de tiempo más pequeños, aumentando notablemente el tiempo de computación. 


\section{RESULTADOS}

Se desarrollo un programa computacional para predecir los tiempos de calentamiento, cuyos parámetros de entrada son la densidad básica de la especie, largo, diámetro de la troza, diámetro del rollizo central, humedad de la madera, temperatura del baño, temperatura inicial y deseada en el rollizo central de la troza. El programa evalúa propiedades térmicas para la humedad y temperatura media de la troza, durante el proceso.

La figura muestra una curva típica de calentamiento de una troza según el modelo de conducción radial.

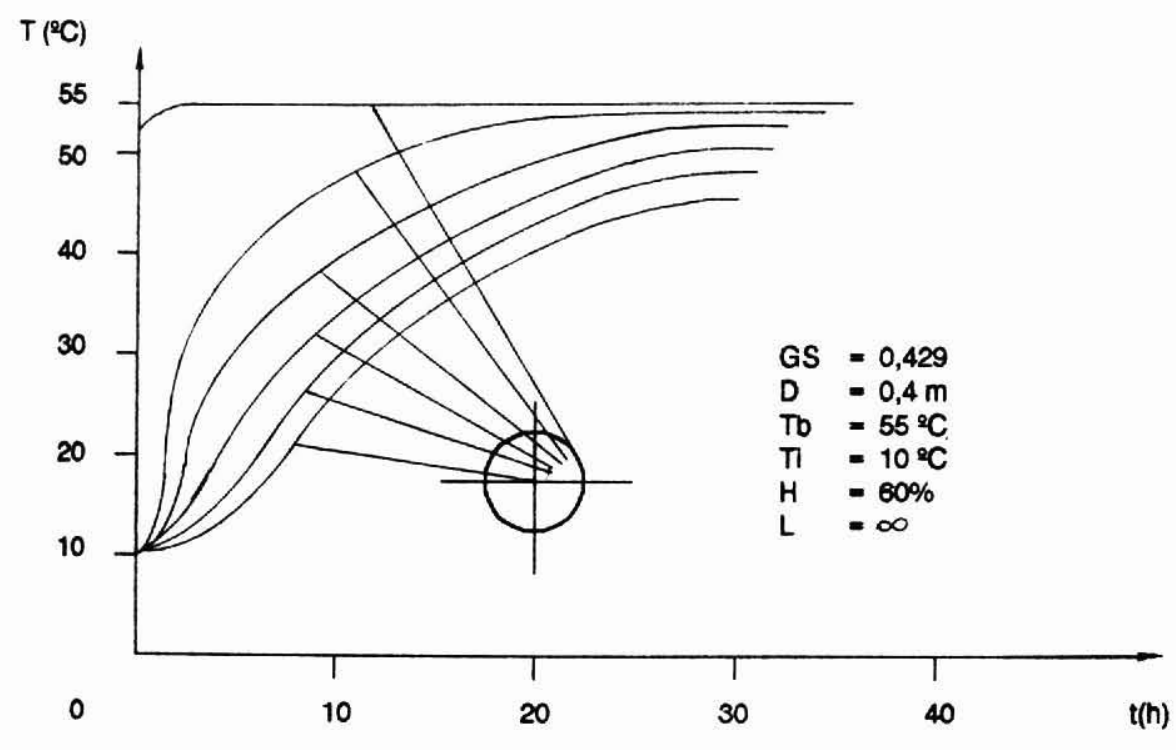

Figura 1.- Campo de temperatura de una troza según modelo unidireccional.

El estudio de la influencia de la anisotropía de la madera en las curvas de calentamiento, se muestra en la figura 2 , que corresponde a la evolución de la temperatura en el tiempo para las fibras que se encuentran en la línea central de una troza de madera de relación 3:1 entre el largo y el diámetro. Las variacioes observadas en los resultados entregados por ambos modelos existen sólo en los extremos de la troza y son inapreciables en el centro geométrico de la misma; así, el tiempo de calentamiento se puede predecir asumiendo un medio isotropico, sin cometer errores significativos, cuando la relacion largo-diámetro es la ya citada, situación normalmente observada en la práctica. 


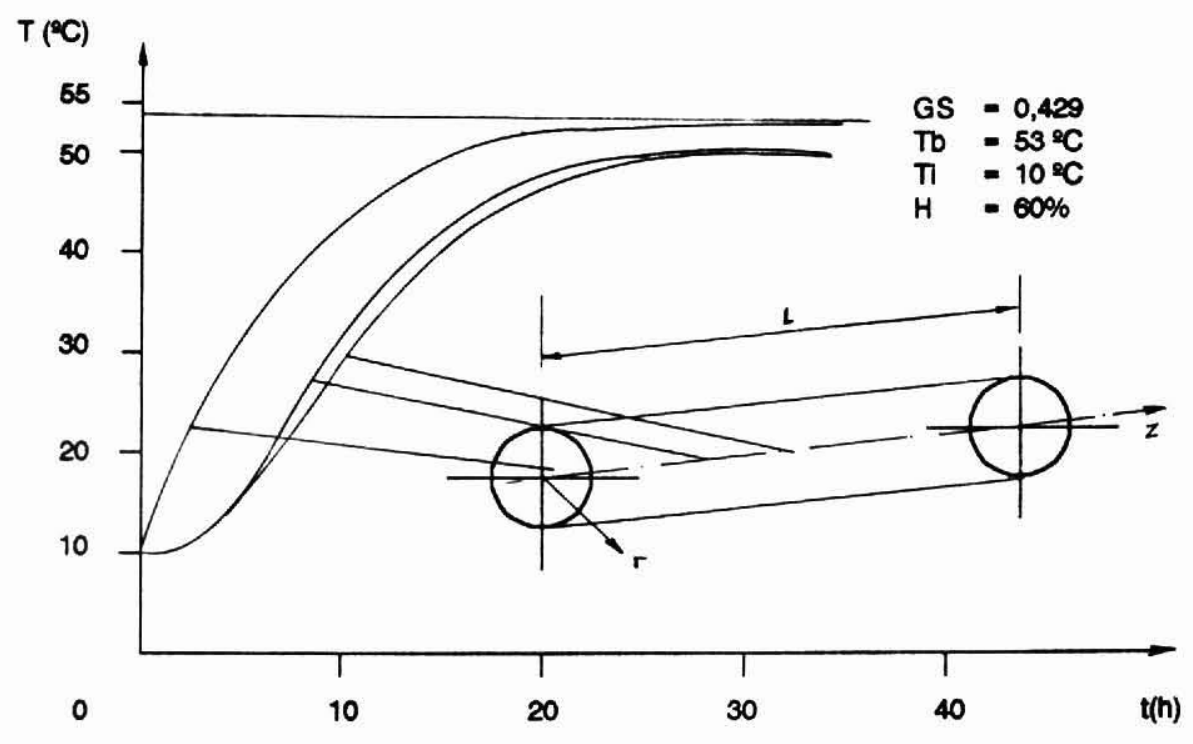

Figura 2.- Evolución de la temperatura en el eje central de una troza considerando la anisotropía de la madera.

Se analizó también la dependencia del tiempo de macerado en función del diámetro de la troza, evidenciándose una relación parabólica entre ambas variables. (figura 3).

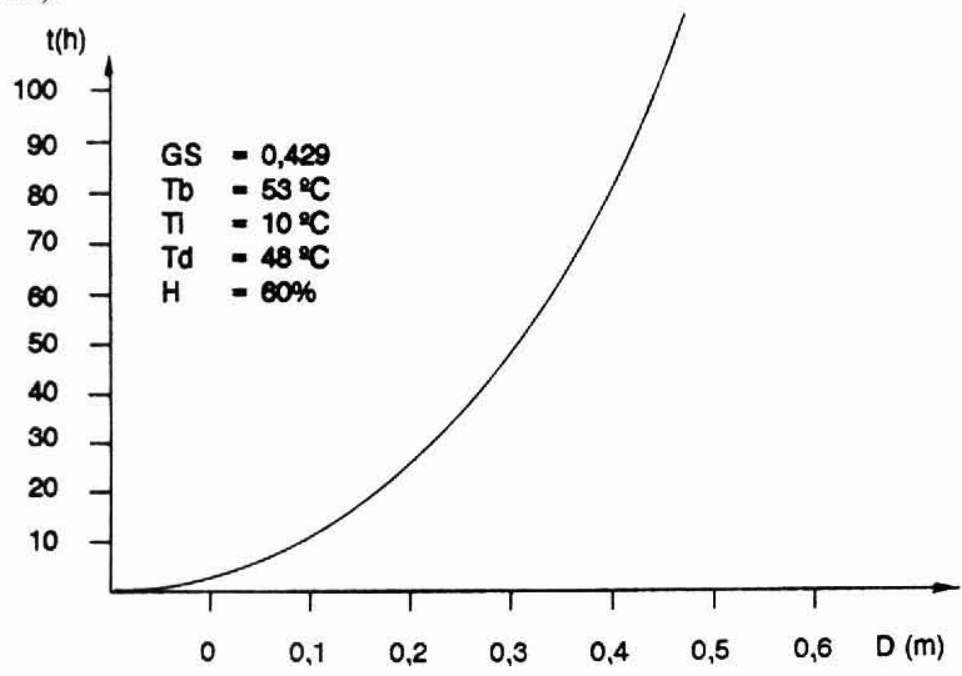

Figura 3.- Tiempo de calentamiento en función del diámetro del rollizo. 
Gran influencia ejerce la temperatura del baño en el tiempo de calentamiento, según lo mostrado en la figura 4 , y es así como aumentos de algunos grados de la temperatura de la pileta pueden reducir significativamente el tiempo necesario de calentamiento.

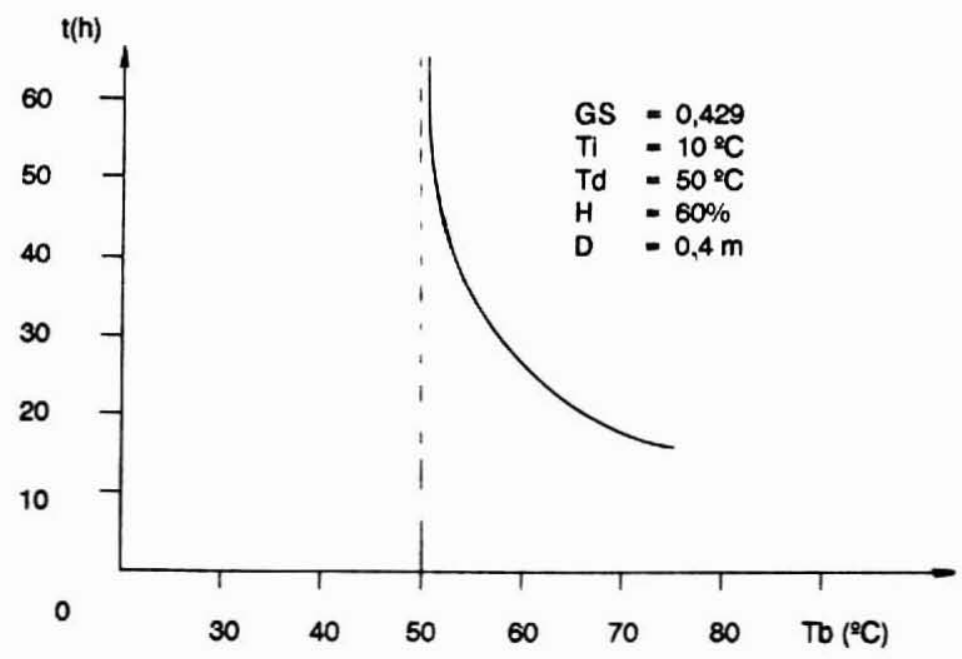

Figura 4.- Tiempo de calentemiento en función de la temperatura del baño.

Las propiedades térmicas de la madera también juegan un rol importante, tal como se muestra en la figura 5.

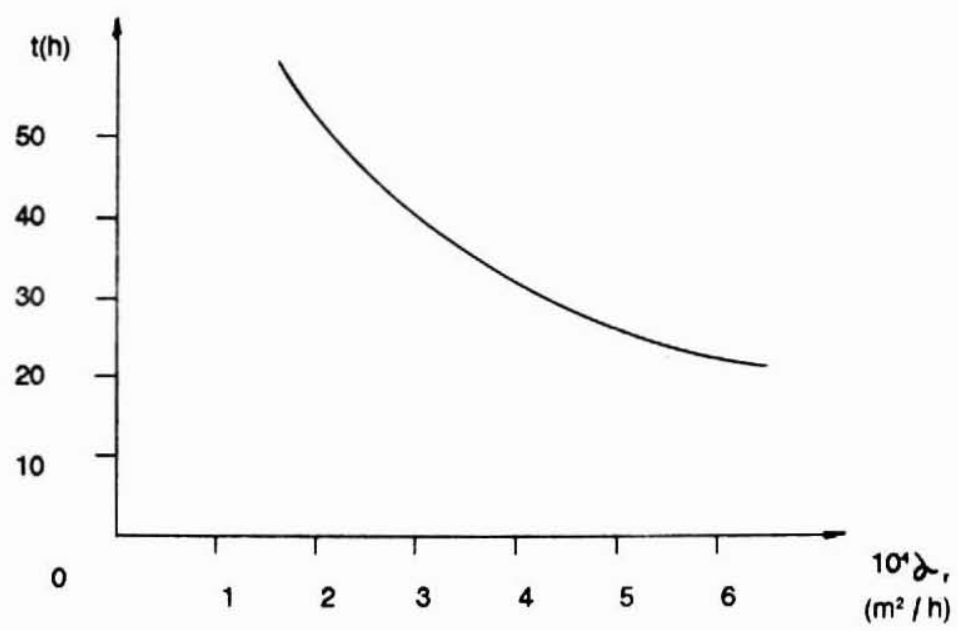

Figura 5.- Tiempo de calentamiento en función de la difusividad térmica. 
Al evaluar la influencia de cada uno de los parámetros que permiten calcular la difusividad se encontró que la densidad básica de la madera no tiene una influencia significativa en el tiempo de calentamiento (Cuadro 1) para alcanzar una temperatura de macerado determinada. No obstante lo anterior, su influencia en la determinación de la temperatura optima de debobinado hace que los tiempos de macerado puedan variar.

Cuadro 1.- Tiempos de macerado para diferentes especies.

\begin{tabular}{|l|c|c|c|c|}
\hline Especie & GS & $\mathrm{Td}\left(\mathrm{C}^{\circ}\right)$ & $\mathrm{Tb}\left(\mathrm{C}^{2}\right)$ & $\mathrm{t}(\mathrm{h})$ \\
\hline Ulmo & 0,537 & 70 & 65 & 38.9 \\
Roble & 0,492 & 70 & 65 & 38,4 \\
Araucaria & 0,483 & 70 & 65 & 38,1 \\
Pino & 0,429 & 70 & 65 & 37,7 \\
Tepa & 0,418 & 70 & 65 & 37,6 \\
Alamo & 0,331 & 70 & 65 & 36,8 \\
\hline
\end{tabular}

$\mathrm{D}=0,4 \mathrm{~m}, \mathrm{Ti}=10^{\circ} \mathrm{C}, \mathrm{H}=60 \%$

La temperatura inicial y el contenido de humedad de la troza son otros parámetros que determinan el valor de las propiedades térmicas y los tiempos de macerado (Cuadro 2 ). Es así que un aumento en el contenido de humedad de la troza y/o bajas temperaturas iniciales de la troza obligan a tener tiempos de macerado mayores.

Cuadro 2.- Tiempos de macerado para diferentes contenidos de humedad y temperatura inicial de la troza.

\begin{tabular}{|c|c|}
\hline $\mathrm{H}(\%)$ & $\mathrm{t}(\mathrm{h})$ \\
\hline 60 & 33,4 \\
80 & 34,9 \\
100 & 35,9 \\
120 & 37,5 \\
\hline
\end{tabular}

\begin{tabular}{|c|c|}
\hline $\mathrm{Ti}\left({ }^{\circ} \mathrm{C}\right)$ & $\mathrm{t}(\mathrm{h})$ \\
\hline 5 & 35,0 \\
10 & 33,4 \\
15 & 31,7 \\
20 & 29,7 \\
\hline
\end{tabular}

$\mathrm{GS}=0,429, \mathrm{D}=0,4 \mathrm{~m}$

$\mathrm{Ti}=10^{\circ} \mathrm{C}$

$\mathrm{GS}=0,429, \mathrm{D}=0,4 \mathrm{~m}$

$\mathrm{Tb}=53^{\circ} \mathrm{C} \quad \mathrm{Td}=48{ }^{\circ} \mathrm{C}$

$\mathrm{H}=60 \%$

$\mathrm{Tb}=53^{\circ} \mathrm{C} \quad \mathrm{Td}=48^{\circ} \mathrm{C}$ 


\section{CONCLUSIONES}

Debido a los altos coeficientes de transferencia de calor por convección entre la troza y el baño de agua caliente $(\mathrm{Bi}>100)$, no se puede esperar una disminución notable en el tiempo de calentamiento al intentar agitar un baño.

La anisotropía de la madera no influye en el tiempo de calentamiento cuando la relación diámetro-largo de la troza es igual o superior a 1:3.

Un calentamiento racionalizado, debe tener en cuenta el tamaño y contenido de humedad de las trozas. Se recomienda seleccionar las trozas de acuerdo a estos parámetros para ser calentadas en pozos diferentes.

El tiempo de calentamiento, puede disminuirse considerablemente a través del control de la temperatura del baño, empleando altas temperaturas al final de la etapa de calentamiento del proceso de macerado.

Se recomienda estudiar el mejor régimen de macerado desde el punto de vista del consumo de energía y los efectos secundarios que se pueden producir en la madera, producto de altas tasas de entrega de calor al emplear temperaturas del baño más altas que las requeridas para el debobinado.

\section{RECONOCIMIENTO}

Este estudio es parte del Proyecto "Estudio de una modelación teórica lineal para el macerado de trozas de madera", inscrito en la Dirección de Investigación y Convenios de la Universidad Austral de Chile. 


\section{REFERENCIAS BIBLIOGRAFICAS}

1.-DEVLIEGER, F. 1986. Técnica de debobinado, Facultad de Ciencias Forestales, Universidad Austral de Chile., 24 p.

2.- FEIHL, O. 1972. Heating frozen and nonfrozen veneer logs. Forest Prod. J. 22(10) : 41-50.

3.- FEIHL, O.; GODIN, V. 1975. Heating veneer logs : a practical guide. Can. Dept. Env., Eastern Forest Prod. Lab., Otawa, Ontario, Canadá. Forestry Tech. Report 9.19 pp.

4.- LUTZ J. F. 1972. Veneer species that grow in the United States. USDA Forest Serv., Forest Prod. Lab., Madison, Wis. Research Paper FPL 167.127 pp.

5.- Mc ADAMS, W. H. 1954. Heat transmission, 3 ed., Mc Graw Hill Book Company, New York.

6.- STEINHAGEN, H. P. 1977. Thermal Conductive properties of wood, green or dry from $-40^{\circ} \mathrm{C}$ to $+100^{\circ} \mathrm{C}$ : a literature review. USDA Forest Serv., Forest Prod. Lab., Madison, Wis General Tech. Report FPL-9 10pp.

7.-STEINHAGEN, H.P.; MYERS, G. E.; KUBLER, H. 1980. Heating time charts for frozen and nonfrozen veneer logs. Forest Prod. J. 30 (40) : 27-37.

8.-STEINHAGEN, H. P. 1986. Computerized finite-difference method to calculate transient heat conduction with thawing. Wood and Fiber Sci. 18 (3) : 460-467.

9.- STEINHAGEN H. P.; LEE, H. W.; LOEHNERTZ, S. P. 1987. LOGHEAT : a computer program for determining log heating times for frozen and nonfrozen logs. Forest Prod. J. 37 (1112) : 60-64. 


\section{NOMENCLATURA}

A Factor de corrección de la difusividad en términos de GS

C Calor específico de la madera $(\mathrm{Kcal} / \mathrm{Kg} \cdot \mathrm{C})$

GS Densidad básica de la madera

D Diámetro de la troza (m)

g Aceleración de gravedad $(\mathrm{m} / \mathrm{s} 2)$

kr Conductividad têrmica radial de la madera $\left(\mathrm{Kcal} / \mathrm{h} \mathrm{m} \mathrm{m}^{\circ} \mathrm{C}\right)$

kz Conductividad térmica axial de la madera ( $\left.\mathrm{Kcal} / \mathrm{h} \mathrm{m}^{\circ} \mathrm{C}\right)$

h Coeficiente convectivo de transferencia de calor troza-agua $\left(\mathrm{Kcal} / \mathrm{h} \mathrm{m} 2{ }^{\circ} \mathrm{C}\right)$

k Conductividad térmica del agua $\left(\mathrm{Kcal} \mathrm{h} \mathrm{m}^{\circ} \mathrm{C}\right)$

L Longitud de la troza (m)

M Parámetro adimensional de estabilidad en método numérico

T Temperatura de la troza $\left({ }^{\circ} \mathrm{C}\right)$

$\mathrm{Tb}$ Temperatura del baño $\left({ }^{\circ} \mathrm{C}\right)$

Td Temperatura deseada para el debobinado $\left({ }^{\circ} \mathrm{C}\right)$

$\mathrm{Ti}$ Temperatura inicial de la troza ( $\left.{ }^{\circ} \mathrm{C}\right)$

$\mathrm{Ti}^{\mathrm{n}} \quad$ Temperatura en el nodo i para el tiempo $\mathrm{n}\left({ }^{\circ} \mathrm{C}\right)$

t Tiempo (h)

$r \quad$ Posición radial en la troza (m)

R Radio de la troza (m)

ej Vector unitario de la dirección j

Pr Número adimensional de Prandtl

$\mathrm{Bi} \quad$ Número adimensional de Biot

Gr Número adimensional de Grashof

$\mathrm{Nu} \quad$ Número adimensional de Nusselt

$\mathrm{H}$ Contenido de humedad de la madera (\%)

$\mathrm{Z}$ Posición longitudinal en la troza (m) 


\section{SIMBOLOS GRIEGOS}

$\alpha 0=$ Difusividad térmica de la madera a $0^{\circ} \mathrm{C}(\mathrm{m} 2 / \mathrm{h})$

$\alpha_{\mathrm{r}}=$ Difusividad térmica radial de la madera $(\mathrm{m} 2 / \mathrm{h})$

$\alpha_{z} \quad=$ Difusividad térmica axial de la madera $(\mathrm{m} 2 / \mathrm{h})$

B = Coeficiente de expansión volumétrica del agua $(1 / \mathrm{K})$

$\rho \quad=$ Densidad aparente de la madera $(\mathrm{Kg} / \mathrm{m} 3)$

$\Delta_{r} \quad=$ Tamaño de un nodo en la dirección radial $(\mathrm{m})$

$\Delta \mathrm{l}=$ Intervalo de tiempo (h)

$\nabla \quad=$ Operador gradiente

$\nabla \cdot \quad=$ Operador divergencia

v $\quad=$ Viscosidad cinemática del agua $(\mathrm{m} 2 / \mathrm{s})$ 